\title{
On the mechanistic origins of maximum strength in nanocrystalline metals
}

\author{
Ankit Gupta (i) ${ }^{1}$, Jacob Gruber ${ }^{1}$, Satish S. Rajaram (iD) ${ }^{2}$, Gregory B. Thompson ${ }^{3}$, David L. McDowell ${ }^{4}$ and Garritt J. Tucker (iD) ${ }^{1 凶}$
}

The maximum strength of polycrystalline metals/alloys has been suggested to occur at nanoscale grain sizes where the governing deformation mechanism transitions from dislocation plasticity to grain boundary mediated deformation. Despite tremendous progress recently uncovering links between transitions in nanoscale mechanisms and peak strength, the scientific literature is mostly devoid of any quantitative support, owing to the difficulty in measuring the resolved contribution of individual mechanisms to microstructural strain accommodation. In this study, the contribution of individual nanoscale mechanisms to the overall deformation of nanocrystalline $\mathrm{Ni}$ is calculated from atomistic simulations leveraging continuum-based kinematic metrics to compute mechanistic contributions to microstructural strain. By employing such a quantitative approach to analyze deformation behavior, it is shown that the realization of maximum strength in nanocrystalline metals corresponds to a grain size regime where the operative nanoscale mechanisms transition, and are thus equally competing to accommodate strain. However, the transition occurs between intergranular and intragranular mediated mechanisms, as it is found that dislocation plasticity alone is not the governing mechanism at all grain sizes above the peak strength regime.

npj Computational Materials (2020)6:153; https://doi.org/10.1038/s41524-020-00425-0

\section{INTRODUCTION}

Materials scientists have long sought to understand and control functional properties, such as mechanical strength, of materials through fundamental knowledge of chemistry and structure (from atoms to the continuum). In the realm of metals and metallic alloys, a significant number of efforts over the past half century have uncovered a seemingly ubiquitous trend regarding increases in mechanical strength and hardness with decreasing average grain size of a polycrystal-the so called 'Hall-Petch' relationship $^{1,2}$. The hardening trend continues well into the nanocrystalline $(\mathrm{NC})$ regime (grain size $<100 \mathrm{~nm})^{3-5}$, with a decrease in slope of the Hall-Petch relationship reported in this regime ${ }^{6,7}$. However, the trend appears to reverse below a critical grain size $(<20 \mathrm{~nm})$. Softening in the strength and hardness of NC materials at such small grain sizes is observed in several experimental studies and also supported by Molecular Dynamics (MD) simulations ${ }^{8-12}$. The maxima in the strength of NC metals were typically observed in a grain-size regime of $10-20 \mathrm{~nm}$ in these studies. The softening regime is generally attributed to a change in the governing deformation mechanism from dislocation-based to grain boundary (GB) mediated deformation because of high interfacial volume fraction and minimal concentration of existing intragranular defects at the smallest of grain sizes ${ }^{10,13-16}$. It is further argued that the grain-size where the transition in the governing deformation mechanism occurs, should correspond to maximum strength ${ }^{17-20}$. The peak strength in several theoretical models is in fact defined by the cross-over between the scaling laws for the strength of NC materials in the softening and the hardening regimes ${ }^{21}$. Comprehensive reviews underlining the current state and the existing gaps in the understanding of mechanical behavior of NC materials are available in the scientific literature $^{22-24}$.
One major gap is regarding the present understanding of the role of individual mechanisms in the deformation of NC materials, which stems predominantly from a qualitative analysis of the mechanical behavior, as pointed out by Spearot et $\mathrm{al}^{22}$. In the majority of seminal studies, the operative nanoscale deformation mechanisms in disparate grain size regimes were established mostly from the structural characterization of NC samples by roughly observing the amount of dislocation slip or twinning in the deformed structures ${ }^{8,9,16,18-20}$. In a few MD studies, the governing deformation mechanisms were at most suggested based on indirect evidences, such as the number (or fraction) of atoms participating in a given mechanism ${ }^{25}$. Yet the relative contributions of individual mechanisms (e.g., dislocation slip, twinning, GB sliding/migration, etc.) towards the overall deformation should ideally be determined by the fraction of strain accommodated by each of these mechanisms during deformation, relative to the total strain accommodation within the material. Quantification of the governing deformation mechanisms directly from the strain accommodated by individual mechanisms is limited to a very few studies ${ }^{26-29}$. The quantification of deformation mechanisms is necessary for verifying the notion that the maximum strength corresponds to a grain size at which the governing deformation mechanism transitions from dislocation to GB mediated processes. Some previous works have quantified the mechanistic crossover based on inflections observed in additional mechanical properties, such as plastic strain rate ${ }^{17}$, activation volume $^{30,31}$, activation enthalpy ${ }^{31}$; or from atomic fractions of different defect structures ${ }^{32}$. However, the notion relating the maximum strength to the mechanistic cross-over, has not been verified directly from the strain accommodated by individual mechanisms, in any previous study. The only such investigation, to the best of the authors' knowledge, was presented by Vo et al. ${ }^{33}$,

\footnotetext{
${ }^{1}$ Colorado School of Mines, Mechanical Engineering, Golden, CO 80401, USA. ${ }^{2}$ Department of Mechanical Engineering, Drexel University, Philadelphia, PA 19104, USA. ${ }^{3}$ Department of Metallurgical and Materials Engineering, University of Alabama, Tuscaloosa, AL 35487, USA. ${ }^{4}$ Department of Mechanical Engineering, Georgia Institute of Technology, Atlanta, GA 30332, USA. ${ }^{\circledR}$ email: tucker@mines.edu
} 
although their discussion was mainly focused on the plastic component of strain.

This study investigates the mechanical behavior of $\mathrm{NC} \mathrm{Ni}$ under uniaxial tension as a function of average grain size using MD simulations. In contrast to the majority of previous investigations, we employ a set of post-processing metrics, based on continuum mechanics, to calculate the contribution of various mechanisms to the overall deformation by resolving the associated fraction of total strain accommodation to individual operative mechanisms during deformation. The mechanism whose contribution to total strain accommodation is greater than $50 \%$, is identified as the governing deformation mechanism. This quantitative approach reveals that while GB-based mechanisms govern the mechanical response during plastic flow in the softening regime, in agreement with previous works, dislocation-mediated plasticity by itself is not the governing deformation mechanism in the hardening regime at grain sizes below $20 \mathrm{~nm}$. Instead we show it is the collective contribution of all intragranular deformation mechanisms that governs the overall response of NC structures in this part of the hardening regime. Importantly, it is also shown that the maximum strength (using calculated flow stress) occurs within a range of grain size at which the governing deformation mechanism transitions from intergranular (i.e., GB-mediated) to intragranular (IG)-based mechanisms. However, these intragranular mechanisms are not solely defined by dislocation slip, as suggested in past studies, but also include contributions from deformation twinning and elastic lattice deformation.

The maximum strength corresponding to the grain size regime at which the governing mechanism transitions, is characterized by an equal accommodation of the total strain between intergranular and intragranular mechanisms during flow. In other words, the dominant deformation mechanisms are equally competing with each other to accommodate microstructural strain. The validity of the notion relating the maximum strength to an equal competition of nanoscale mechanisms (IG versus GB), is further examined individually for elastic and plastic components of strain and in the context of potential limitations or artifacts of MD simulations, such as the high strain rate. Finally, the mechanical behavior of a $\mathrm{NC} \mathrm{Ni}$ alloy is presented, to further highlight the significance of the findings of this study with respect to shifting the strongest grain size regime in NC alloys by varying the contributions of underlying mechanisms, where the trends agree well with available experimental data.

\section{RESULTS AND DISCUSSION}

Mechanical behavior of $\mathrm{NC} \mathrm{Ni}$

In order to probe the mechanical behavior as a function of grain size, we performed MD simulations on $3 D$ atomistic structures of $\mathrm{NC} \mathrm{Ni}$ with average grain sizes ranging from $6 \mathrm{~nm}$ to $20 \mathrm{~nm}$. NC structures were loaded uniaxially at $300 \mathrm{~K}$ to a total tensile strain of $12 \%$ at constant rate of $10^{8} \mathrm{~s}^{-1}$. More details regarding the generation and tensile testing of these structures can be found in the "Methods" section. Figure $1 \mathrm{a}$, b shows the average stress-strain response of different samples with varying average grain size. All curves show an initial elastic deformation regime, where stress increases linearly with strain. The initial elastic stiffness (slope of the curve below $1 \%$ strain) increases with increasing grain size, in agreement with reported experimental trends ${ }^{34,35}$. Since MD simulations are at much higher strain rates then what is typically achieved experimentally, such agreement adds confidence in linking simulations to experimental observations. Beyond $1 \%$ strain, the curves begin to deviate from the apparent linear stressstrain behavior. The deviation from linearity is generally attributed to activation of several microplasticity events in NC structures ${ }^{27,36-38}$. The linear regime ends completely around $3-4 \%$ strain in all grain size structures after macroscopic yielding of the samples. The grain size dependent yielding behavior of NC metals including the onset of plasticity is discussed in-depth in our previous work ${ }^{36}$. Gradual softening can be observed in the stress response prior to flow, especially for large grain size structures. In this work, we mainly focus on the deformation behavior in the flow regime beyond 5\% strain, when most of the strain accommodation becomes plastic in nature. The flow stress was calculated by averaging stress values in this regime between $5-12 \%$ strain.

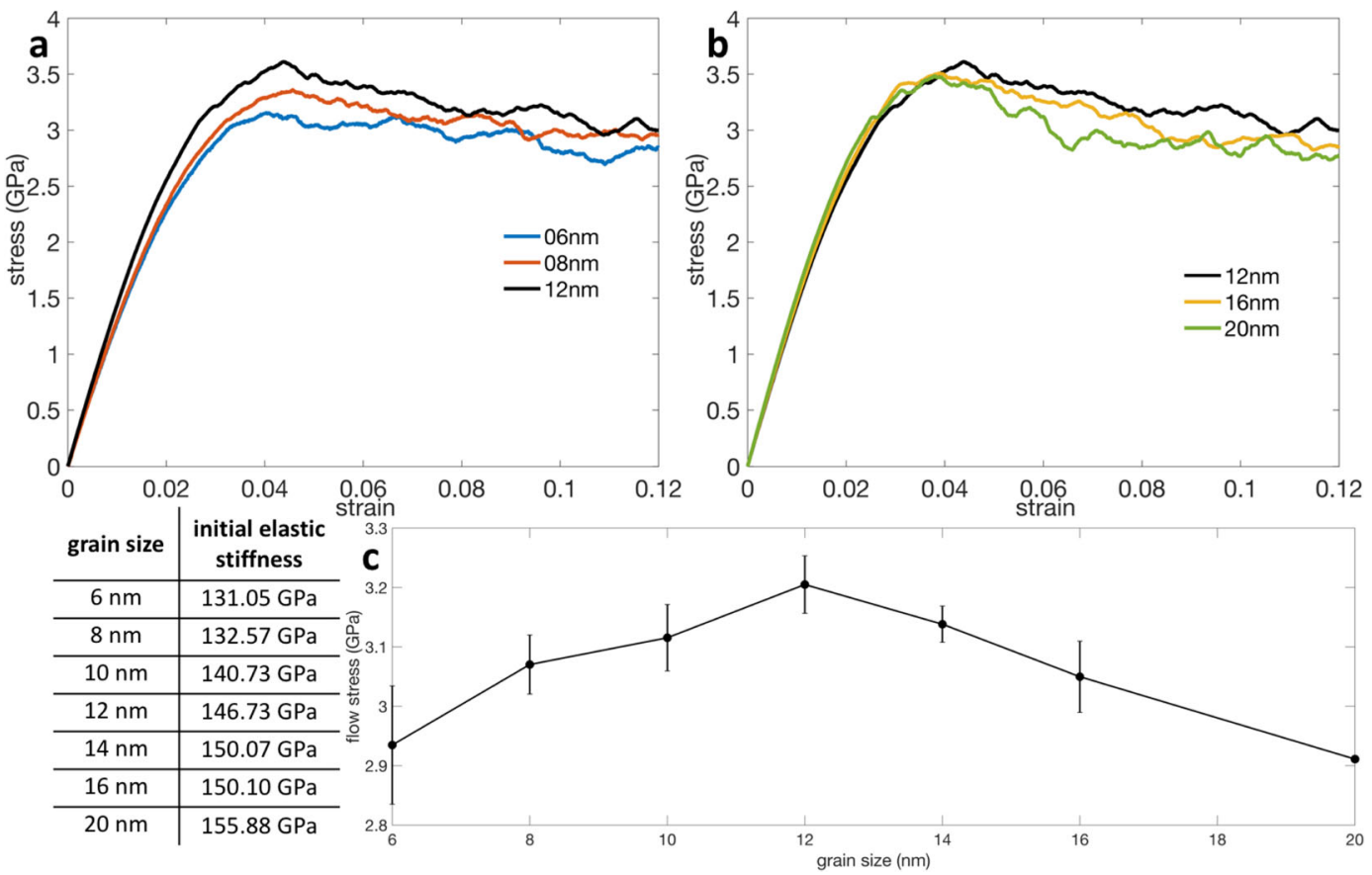

Fig. 1 Mechanical behavior of NC Ni. Stress-strain curves obtained from the uniaxial tension MD simulations of NC structures with average grain size ranging from (a) 6-12 nm and (b) $12-20 \mathrm{~nm}$ (initial elastic stiffness values are listed in the table). (c) Variation of flow stress as a function of average grain size of NC structures. 


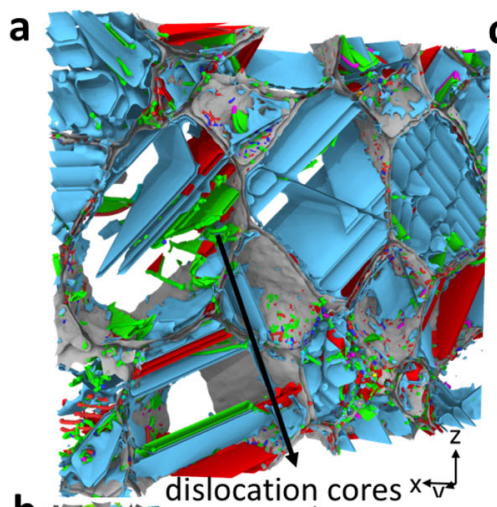

b

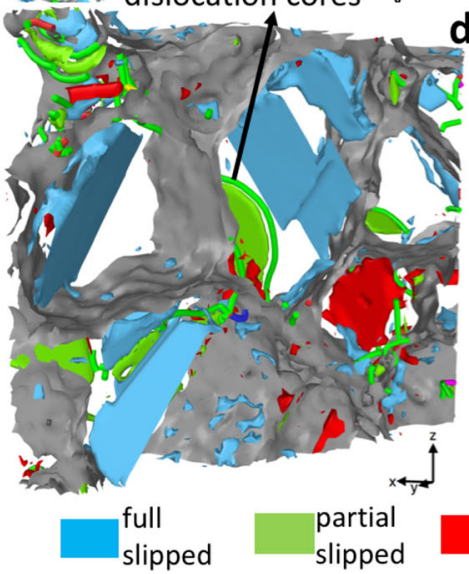

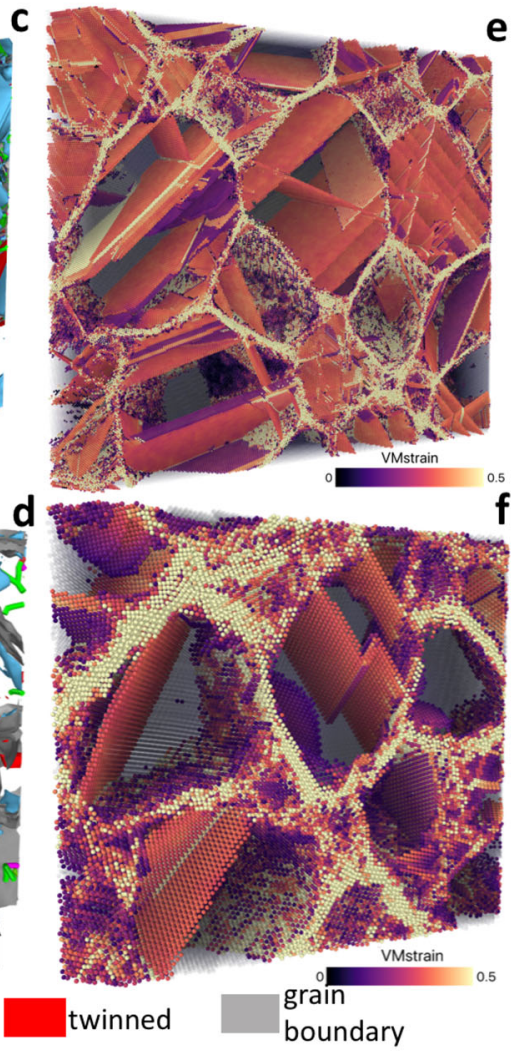
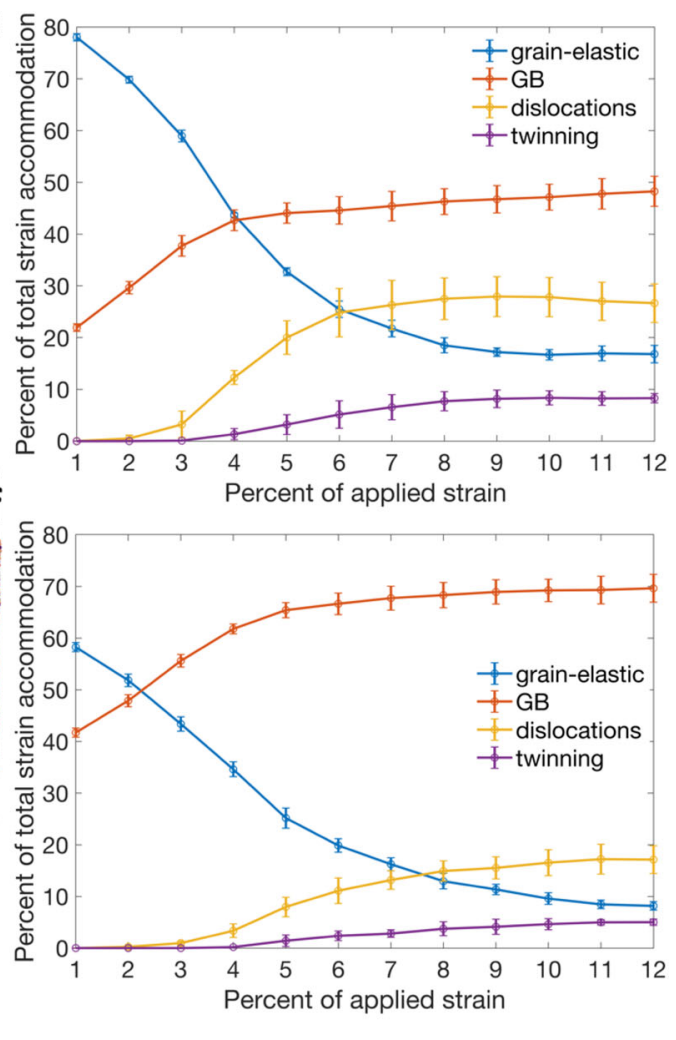

Fig. 2 Deformation mechanisms in NC Ni. Slices through the NC Ni structures of average grain size of $16 \mathrm{~nm}(\mathbf{a}, \mathbf{c})$ and $6 \mathrm{~nm}(\mathbf{b}$, d), with atoms colored according to the identified nanoscale deformation mechanisms (a, b), blue: full slip, green: partial slip, red: twinning, gray: GBs, and von Mises strain accommodation (c, d) at $12 \%$ applied strain. Percentages of total strain accommodation by individual deformation mechanisms, as a function of applied strain, during uniaxial loading of (e) $16 \mathrm{~nm}$ and (f) $6 \mathrm{~nm}$ grain size NC structures.

Figure 1c shows the variation of flow stress as a function of average grain size obtained from the tensile loading simulations of $\mathrm{NC} \mathrm{Ni.} \mathrm{Each} \mathrm{data} \mathrm{point} \mathrm{is} \mathrm{the} \mathrm{average} \mathrm{of} \mathrm{the} \mathrm{flow-stress} \mathrm{values}$ calculated from five different simulations at every grain size and the error bars represent the standard deviation in the values. It should be noted here that the grain sizes mentioned here and in the rest of the study, are initial grain sizes of the NC structures before tensile loading. Some grain growth is noticed after tensile loading; the extent of which decreases with increasing grain size. After $12 \%$ applied strain, the average grain size increased by $1.09 \mathrm{~nm}$ and $0.41 \mathrm{~nm}$ for NC Ni structures with initial grain sizes of $6 \mathrm{~nm}$ and $20 \mathrm{~nm}$, respectively. As clearly shown, the flow stress increases when the grain size decreases from $20 \mathrm{~nm}$ to $12 \mathrm{~nm}$ (i.e., the hardening regime) but then decreases when the grain size reduces below $12 \mathrm{~nm}$ (i.e., the softening regime). In terms of the calculated flow stress, the $12 \mathrm{~nm}$ grain size structure is found to be the strongest. As noted above, previous studies have also reported the presence of hardening and softening regimes in the strength versus grain size plots of several metals with a maximum appearing in the NC regime ${ }^{23}$. We further apply a quantitative analysis to investigate the competition/cooperation of the nanoscale deformation mechanisms to accommodate microstructural strain at various grain sizes. A set of recently developed postprocessing tools ${ }^{39}$ were employed to distinguish individual mechanisms operating in NC structures and calculate their relative contributions to microstructural strain accommodation.

\section{Deformation mechanisms in $\mathrm{NC} \mathrm{Ni}$}

Atoms participating in various deformation mechanisms (i.e., dislocation slip, deformation twinning, elastic lattice strain, and GB deformation) were identified based upon the calculated slip vector, structural environment and kinematic signatures of deformation (see "Methods" section). Figure 2a, b shows atoms sheared by different deformation mechanisms through slices of NC Ni structures of $16 \mathrm{~nm}$ and $6 \mathrm{~nm}$ grain size, respectively, at the end of tensile loading simulation, i.e., 12\% applied strain. The complete evolution of these structures with strain is presented in Supplementary Movies 1 and 2, respectively. Atoms that deformed by either dislocations (partial or full), twinning or GB-based mechanisms are shown in these structures. The remaining grain atoms (rendered invisible) deformed in an elastic manner. The degree of twinning appears to be small in both grain size structures. The $16 \mathrm{~nm}$ grain structure lying in the hardening regime of the flow stress-grain size plot, reveals more dislocation activity than the $6 \mathrm{~nm}$ NC structure, which lies in the softening regime. In many earlier seminal studies ${ }^{9,19,20}$, dislocations and GBs were established as the governing deformation mechanisms in the hardening and the softening regimes, respectively, based solely on such qualitative analysis of deformed images or micrographs in the two regimes and roughly comparing the amount of defect structures. However, as mentioned earlier, it is not the number of atoms in each defect or mechanism, but the total strain accommodated by those atoms participating in a given mechanism, which should ideally determine the contribution of that mechanism to the overall deformation. Therefore, in order to calculate the amount of strain accommodated within individual mechanisms, the strain tensors of atoms in the NC structure were computed using continuum-based kinematic metrics $26,28,40,41$ by mapping changes in local atomic neighborhoods during deformation. From the calculated atomic strain tensors and the identification of atoms participating in various deformation mechanisms, the fraction of strain accommodated by individual mechanisms 

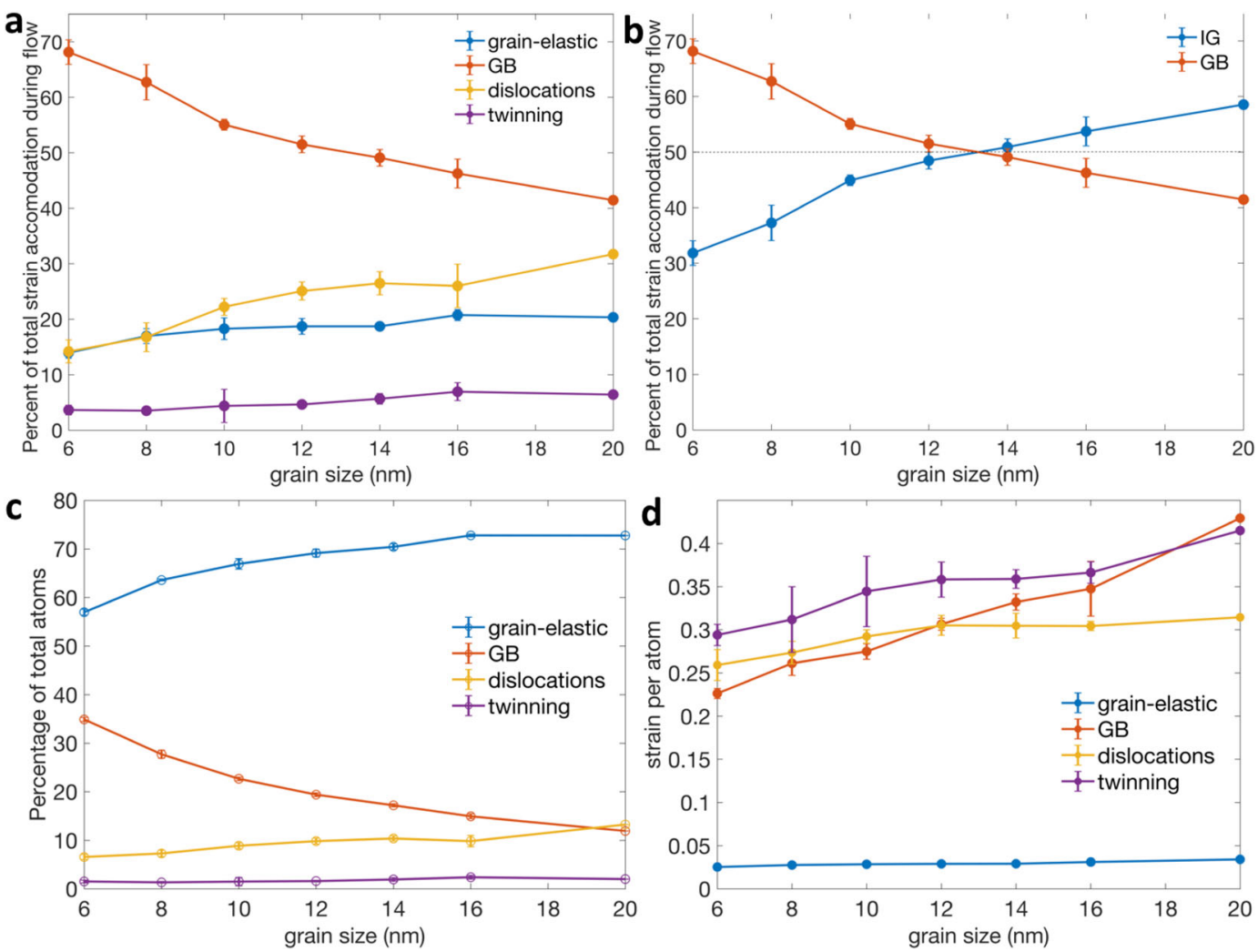

Fig. 3 Competition of deformation mechanisms. Variation of percentages of total strain accommodation by (a) individual mechanisms and (b) intragranular (IG) versus intergranular (GB) mechanisms during flow; and variation of (c) percentages of total atoms and (d) strain-per atom associated with individual mechanisms during flow, as a function of average grain size of NC Ni structures.

was obtained. Further details can be found in the "Methods" section.

In Fig. 2c, d, the slices of $16 \mathrm{~nm}$ and $6 \mathrm{~nm}$ average grain size structures are colored according to the von Mises (VM) strain in atoms, obtained from the atomic strain tensors calculated at $12 \%$ applied strain. In particular, atoms associated with the plastic deformation mechanisms show large VM strain. Atomic VM strain is generally higher in the GB regions than the twinned or slipped intragranular regions. The remaining atoms that underwent elastic deformation can be seen as the black shaded regions with small VM strain values. The percentages of total strain accommodation by individual mechanisms are plotted in Fig. 2e, $\mathrm{f}$, as a function of applied strain for the $16 \mathrm{~nm}$ and $6 \mathrm{~nm}$ grain size structures, respectively. Plotted values are averages of the results from five different simulations for each grain size. Similar plots for rest of the grain sizes are shown in supplementary Fig. S1. In both grain size structures, total tensile strain is accommodated in a shared manner between GBs, dislocation slip, twinning, and elastic deformation of grains (grain-elastic). Most of the strain is accommodated by GBs and elastic deformation of grains during initial loading, corresponding to the initial linear elastic regime in the stress-strain curves. Importantly, it will be shown in the next section, where the elastic and plastic components of strain are delineated, that most of the strain accommodated by GBs during this early stage of loading is also elastic in nature. The ratio of strain accommodation between grains to GBs during the initial linear elastic regime is higher in the $16 \mathrm{~nm}$ grain size than the $6 \mathrm{~nm}$ gain size structure.

The percentages of total strain accommodated by dislocations and twinning mechanisms start to rise around 3-4\% strain in both grain size structures (Fig. 2e, f) representative of yielding and aligning with the end of the initial elastic regime. While the relative contributions of various mechanisms change rapidly during the initial elastic regime and yielding, they eventually plateau during the flow regime as also reflected in flattening out of the stress response beyond 5\% strain. In this flow regime, the total contribution of intragranular plastic deformation mechanisms (dislocation slip + twinning) towards strain accommodation is greater than the contribution of elastic deformation of grains, with dislocation slip accommodating more strain than twinning. The percentage of total strain accommodated by the GBs also increases during the flow regime where the majority of the strain accommodation is also plastic in nature. It is seen for the $6 \mathrm{~nm}$ case, most of the strain (around 70\%) is accommodated by the GBs during flow, indicating that GBs can be quantitatively identified as the governing deformation mechanism for this grain-size in the softening regime. Although the contribution of dislocation mechanisms to strain accommodation during flow is higher in the $16 \mathrm{~nm}$ case (Fig. 2e), the actual percentage is only around $25 \%$. Since most of the strain during flow is not accommodated by dislocation slip, dislocation plasticity cannot be suggested as the governing deformation mechanisms for the $16 \mathrm{~nm}$ grain-size structure in the hardening regime. This is found to be true for higher grain sizes (i.e., up to $20 \mathrm{~nm}$ ) as well.

To more clearly show the effect of NC grain size on the governing deformation mechanism, averages of the percentages of total strain accommodation by individual mechanisms during the flow regime $(5-12 \%$ strain) of mechanical loading were calculated for every grain size structure and plotted in Fig. 3a. For a single grain size, the plotted value is the mean of the five different simulations, and the error bar represents the standard deviation. Since most of the strain $(>50 \%)$ during flow is accommodated by the GBs for grain sizes lying in the softening regime $(6-12 \mathrm{~nm}), \mathrm{GB}$ mediated deformation processes (e.g., sliding, migration, atomic shuffling, etc.) can be established as the governing deformation mechanism in this regime. However, 
even for the largest grain size structure (i.e., $20 \mathrm{~nm}$ ) simulated in the hardening regime, the percentage of total strain accommodated by dislocation slip during flow is just 30\% (Fig. 3a). Based upon the fraction of strain accommodation, dislocation plasticity by itself cannot be identified as the governing deformation mechanism in the hardening regime for structures with grain size up to $20 \mathrm{~nm}$. While dislocation slip will eventually govern the deformation at larger grain sizes $(>20 \mathrm{~nm})$, it is not found to be necessarily true of all grain sizes in the hardening regime. Moreover, it is generally suggested in the literature that NC metals should exhibit maximum strength at a grain size where the governing deformation mechanism transitions from dislocation slip to GB-mediated processes ${ }^{17,18}$. Our results do not support this conjecture, as the strongest grain size calculated in this study is $12 \mathrm{~nm}$, but according to Fig. 3a, the transition between dislocations and GB mechanisms will occur at a grain size greater than $20 \mathrm{~nm}$. Instead of comparing the contribution of GB mechanisms with only dislocation mechanism, it is likely more logical to compare with the contribution of all intragranular mechanisms because GB mechanisms also encompass the contributions of a variety of deformation processes including elastic contributions.

The contributions or the percentages of total strain accommodation during flow within twinning, dislocation, and grainelastic mechanisms were summed up to represent the total contribution of intragranular (IG) deformation. The intragranular contributions are compared with the contributions of $G B$ mechanisms for different grain sizes in Fig. 3b. Clearly, GBmediated mechanisms accommodate more strain during flow than intragranular mechanisms in the softening regime $(6-12 \mathrm{~nm}$ grain size). Whereas in the hardening regime $(14-20 \mathrm{~nm}$ grain size), the percentage of total strain accommodation during flow within intragranular mechanisms is higher. These data and trends suggest that all intragranular mechanisms acting together, not just dislocation slip or twinning by itself, govern the mechanical response of NC metals in the hardening regime. Figure $3 \mathrm{~b}$ further demonstrates that the cross-over between the contributions of GB-based (i.e., intergranular) and intragranular mechanisms occurs in the 12-14 nm grain size regime, around which the flow stress reaches a maximum. It indicates that the strongest grain size regime does correspond to a transition in the governing deformation mechanism but from intergranular (i.e., GB-based) to intragranular. The latter includes the contribution of deformation twinning and elastic lattice deformation, not just dislocation slip as previously suggested in the literature. At grain sizes where this transition occurs $(12-14 \mathrm{~nm})$, the total strain is equally accommodated between intergranular and intragranular mechanisms during flow (Fig. 3b). Therefore, these results indicate that maximum strength in NC metals is observed at a grain-size regime where intragranular and GB-based deformation mechanisms are equally competing to accommodate strain during the flow regime.

As discussed before, some previous studies have also quantified the mechanistic crossover in terms of percentage/fraction of atoms participating in different mechanisms. In order to present such analysis, the percentages of total atoms participating in individual mechanisms are calculated from the metric code. The values are averaged over the flow regime and plotted as function of grain size in Fig. 3c. It can be observed from the figure that grain-elastic contributes the most, in terms of percentage of atoms, across all grain-sizes. Hence, no cross-over is noticed between the percentages of total atoms participating in intragranular and GB mechanisms at any grain size. It should also be noted that a cross-over is observed between the percentage of atoms in dislocation and GB mechanisms at a grain size regime of 18-20 nm, however, it does not correspond to the strongest grain size regime. The cross-over regime does not shift much even when the percentages of twinned and dislocation atoms are combined. Therefore, it can be said that the maximum strength in NC metals is only observed at a grain size regime, where the total strain accommodated in the intragranular and GB mechanisms during flow, are equal. The strain-per-atom associated with each mechanism, obtained simply up on dividing the total strain by number of atoms in a given mechanism, is averaged over the flow regime and plotted in Fig. 3d, as function of grain size. As expected, intragranular plastic deformation mechanisms (i.e, dislocations and twinning) accommodate more strain-per-atom as compared to the elastic deformation of grain, across all grain sizes. The strain-per-atom associated with GB mechanisms is also higher and comparable to that of intragranular plastic mechanisms, however, the former also has some elastic contribution. It might then be informative to delineate the elastic and plastic components of strain accommodated in intragranular and GB mechanisms and check if the notion relating the maximum strength to an equal competition of nanoscale mechanisms, holds independently for each component.

\section{Elastic and plastic components of strain}

Plastic strain was computed from the atomic strain tensors after dynamically relaxing the deformed NC structures at several strain increments to a zero-stress configuration (see "Methods" section). Elastic strain was obtained by subtracting the plastic strain from the total applied strain, calculated from the atomic strain tensors in the deformed NC structures before relaxation. Elastic strain can also be calculated from the stress-strain response of the sample during uniaxial loading, simply as $\sigma$ divided by $E^{33}$. Here, $\sigma$ is the stress value at the corresponding strain level and $E$ is the initial elastic stiffness of the sample. Figure $4 a$ plots the elastic component of strain (as percent total strain), obtained from the atomic strain tensors, as a function of applied strain for the $6 \mathrm{~nm}$, $12 \mathrm{~nm}$, and $16 \mathrm{~nm}$ grain size $\mathrm{NC} \mathrm{Ni}$ structures. The elastic component of strain, calculated from the sample's stress strain response, is also shown for the $6 \mathrm{~nm}$ grain size structure. The elastic strain calculated from the kinematic metrics at the atomic scale matches well with the values obtained from the macroscopic stress-strain response of the simulation cell. It demonstrates the high accuracy of the kinematic metrics, especially the method used to approximate the continuum strain field around each atom.

In addition, the plots (Fig. 4a) show that the elastic strain decreases below $50 \%$ in all grain size structures around $5 \%$ applied strain - signifying the beginning of flow when most of the strain accommodation becomes plastic in nature. Figure $4 b, c$ show percentages of total strain accommodation in intragranular (IG) and intergranular (GB) mechanisms, delineated into plastic and elastic components, as a function of applied strain for the $16 \mathrm{~nm}$ and $6 \mathrm{~nm}$ grain size structures, respectively. Clearly, most of the strain accommodation in both grains and GBs is initially elastic in nature while the plastic strain dominates during the flow regime for the two grain size structures, hence, confirming our previous assertions. However, the elastic component of strain does not completely vanish in the flow regime. It can be observed from these plots that the contribution of elastic strain in the flow regime is higher compared to what is typically observed in coarsegrained metals. The source of relatively large elastic strain during the flow regime is generally the high overall stress required to carry plastic deformation in NC metals. As discussed earlier, the elastic component of strain at any point during the loading is equal to the applied stress $\sigma$ divided by the initial elastic stiffness $E^{33}$. While the variation in elastic modulus can be small, the yield and flow stresses increase significantly as grain size approaches the NC regime ${ }^{38}$, which also results in large amount of elastic strain prior to yielding or flow. Since, after yielding, only small stress relaxations are noticed in the stress-strain curves of NC metals (Fig. 1), they retain majority of the large elastic strain acquired before yielding, during the flow regime as well. Furthermore, the competition between intragranular and 

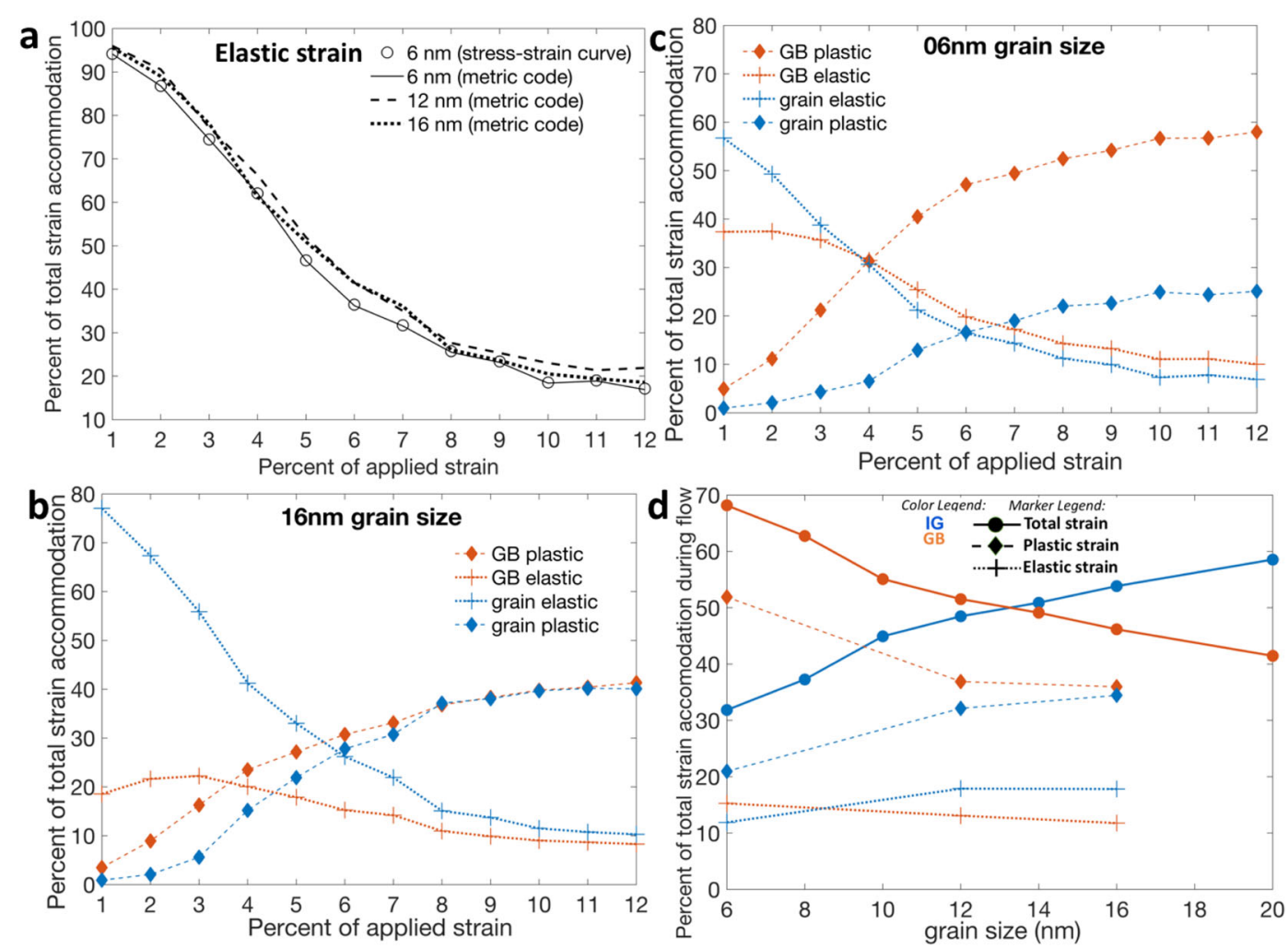

Fig. 4 Elastic versus plastic deformation. (a) Variation of elastic component of strain calculated as percent total strain and obtained from the atomic strain tensors, as a function of applied strain during uniaxial tensile loading of $6 \mathrm{~nm}, 12 \mathrm{~nm}$ and $16 \mathrm{~nm}$ grain size NC Ni structures (elastic strain calculated from the sample's stress strain response is also plotted for the $6 \mathrm{~nm}$ case). Percentages of total strain accommodation by intragranular (IG) and intergranular (GB) mechanisms, delineated into plastic and elastic components, as a function of applied strain for the (b) $16 \mathrm{~nm}$ and (c) $6 \mathrm{~nm}$ grain size structures. (d) Percentages of total strain accommodation by IG and GB mechanisms during flow, delineated into plastic and elastic components, as a function of average grain size of NC Ni structures.

GB-mediated deformation mechanisms in accommodating elastic and plastic deformation can be studied from these plots.

The average contributions of intragranular and GB mechanisms towards total strain accommodation during the flow regime are plotted as a function of grain size in Fig. $4 \mathrm{~d}$. The percentage of total strain accommodation are further broken down into its elastic and plastic components for the $6 \mathrm{~nm}, 12 \mathrm{~nm}$, and $16 \mathrm{~nm}$ grain size structures in order to investigate the competition of deformation mechanisms in the two components individually. As compared to the grain size where the contributions of intergranular and intragranular mechanisms towards total strain accommodation become equal (i.e., $12-14 \mathrm{~nm}$ ), the cross-over between the two mechanisms occurs at a smaller grain size for the elastic component and at a larger grain size for the plastic component. Therefore, we can comment on the notion relating the maximum strength of NC metals to the grain size where intragranular and GB mechanisms contribute equally to strain accommodation during flow; this notion holds for total strain, but not necessarily for its elastic and plastic components individually. It should be noted here that the relative contribution of elastic and plastic components of strain also depends upon the loading strain rate, which is extremely large in MD simulations. Thereafter, it might be interesting to discuss these findings within the scope of artifacts of MD simulations such as high strain rate.

\section{Effect of strain rate}

One of the biggest limitations of MD loading simulations is often the inherent high strain rates encountered during deformation ${ }^{42}$. The simulations described to this point were conducted at a strain rate of $10^{8} \mathrm{~s}^{-1}$. To explore the effect of strain rate on the validity of the mechanistic competition notion discussed above, we also performed uniaxial tensile loading simulations of the $\mathrm{NC} \mathrm{Ni}$ structures of different grain sizes at the strain rate of $10^{10} \mathrm{~s}^{-1}$. Consideration of lower strain rate MD simulations, while desirable, was not feasible with the current computational resources. Figure 5 a shows the stress-strain response during tensile loading of the $16 \mathrm{~nm}$ grain size NC structure at two different strain rates (e.g., $10^{8}$ $s^{-1}, 10^{10} s^{-1}$ ). While the initial slope of the curves in the linear elastic regime is independent of the strain rate, the response diverges significantly as strain increases further. The linear elastic regime extends to larger applied strains at $10^{10} \mathrm{~s}^{-1}$, indicating delayed onset of plasticity at higher strain rate. As a result, the peak in the stress-strain curve appears near $7 \%$ strain at $10^{10} \mathrm{~s}^{-1}$, shifting from $3 \%$ strain at $10^{8} \mathrm{~s}^{-1}$. The peak stress also increases in magnitude at higher strain rate. The stress response eventually flattens out to a steady state flow in the two cases. However, values of both the flow stress and the applied strain where the flow regime begins, are higher at $10^{10} \mathrm{~s}^{-1}$. Similar stress-strain behaviors of NC structures at different MD strain rates were also reported in previous modeling studies $33,42,43$.

The delayed onset of plasticity at higher strain rate is also reflected in Fig. 5b. It plots the percentages of total strain accommodation by individual mechanisms in the $16 \mathrm{~nm}$ grain size structure, as a function of applied strain, at two different rates of $10^{8} \mathrm{~s}^{-1}$ and $10^{10} \mathrm{~s}^{-1}$. Similar plots for rest of the grain sizes are shown in supplementary Fig. S2. The plot shows that most of strain is accommodated in the elastic deformation of grains up to $7 \%$ applied strain during loading at $10^{10} \mathrm{~s}^{-1}$. The contributions of intragranular plastic deformation mechanisms such as dislocation slip and twinning only start to increase after 6-7\% strain at higher strain rate. The delayed yielding further causes the flow regime to shift to higher strain values in the corresponding stress-strain plot 

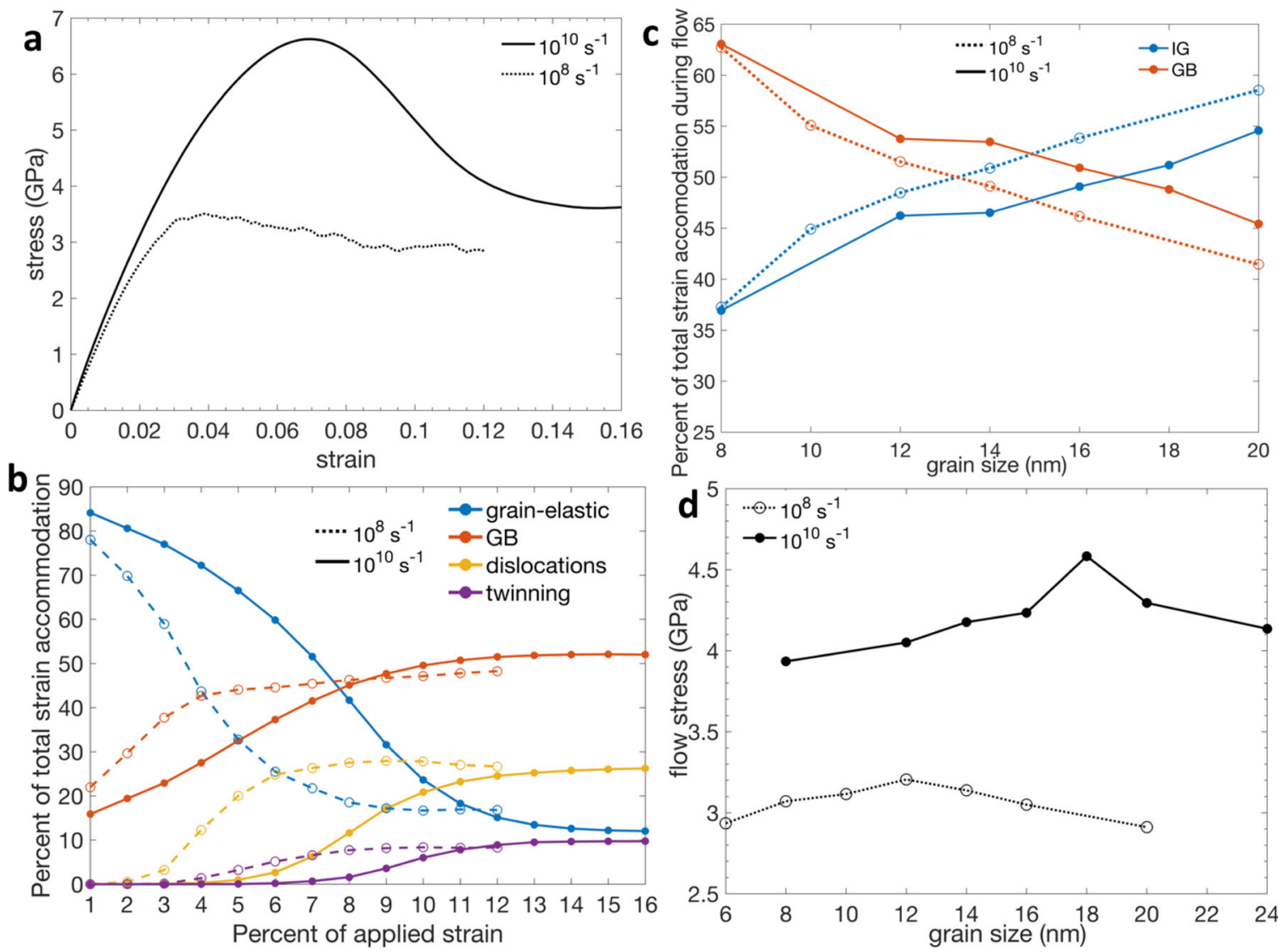

Fig. 5 Effect of strain rate. (a) Stress-strain response of $16 \mathrm{~nm}$ grain size NC Ni structure obtained from the uniaxial tension MD simulations performed at strain rates of $10^{8} \mathrm{~s}^{-1}$ and $10^{10} \mathrm{~s}^{-1}$. (b) Percentages of total strain accommodation by deformation mechanisms, as a function of applied strain, during uniaxial loading of $16 \mathrm{~nm}$ structure at strain rates of $10^{8} \mathrm{~s}^{-1}$ and $10^{10} \mathrm{~s}^{-1}$. Variation of (c) percentages of total strain accommodation by intragranular (IG) and intergranular (GB) mechanisms during flow and (d) flow stress, as a function of average grain size of $\mathrm{NC} \mathrm{Ni}$ structures, obtained from the loading at strain rates of $10^{8} \mathrm{~s}^{-1}$ and $10^{10} \mathrm{~s}^{-1}$.

(Fig. 5a). As seen in Fig. 5b, the contributions of individual mechanisms at $9 \%$ applied strain at $10^{10} \mathrm{~s}^{-1}$ and at $5 \%$ strain at $10^{8} \mathrm{~s}^{-1}$ are similar for the $16 \mathrm{~nm}$ grain size structure. Therefore, the exact strain value, marking the beginning of the flow regime at $10^{10} \mathrm{~s}^{-1}$ strain rate, is taken to be $9 \%$, so that the contributions of deformation mechanisms at the beginning of the flow regime for the two strain rates are comparable. It should also be noted that the NC Ni structures were loaded up to a total tensile strain of $16 \%$ at $10^{10} \mathrm{~s}^{-1}$ strain rate to ensure the same length of the flow regime as the lower strain rate. Figure $5 \mathrm{~b}$ also demonstrates that in the $16 \mathrm{~nm}$ grain structure, the overall contribution of intragranular plastic deformation mechanisms, such as dislocation slip, during flow is smaller at $10^{10} \mathrm{~s}^{-1}$; instead, the percentage of strain accommodated within GB mechanisms increases as compared to their relative contributions at $10^{8} \mathrm{~s}^{-1}$. The reduced contribution and the delayed activation of intragranular plastic deformation mechanisms, particularly dislocation plasticity as observed in this study, can be attributed to the enhanced difficulty of dislocation nucleation and propagation at higher strain rates, as reported in the literature ${ }^{44-46}$.

Similar trends in the variation of the contributions of intergranular (GB) and intragranular (IG) deformation mechanisms during the flow regime, as a function of strain rate, can be observed across all grain sizes of the NC Ni structure in Fig. $5 \mathrm{c}$. The percentages of total strain accommodation at $10^{10} \mathrm{~s}^{-1}$ strain rate, averaged over the flow regime, decrease in general for intragranular mechanisms and increase for intergranular mechanisms across all grain sizes. However, the relative changes in the magnitude are higher for larger grain size structures. As a consequence, the cross-over between the two mechanisms occurs within a grain size regime of $16-18 \mathrm{~nm}$ at $10^{10} \mathrm{~s}^{-1}$. Importantly, the maximum flow stress (plotted in Fig. $5 \mathrm{~d}$ as a function of grain size) also shifts to a larger grain size $(18 \mathrm{~nm})$, corresponding to the grain size regime where the governing deformation mechanism once again transitions. Therefore, it can be concluded that the notion relating the maximum strength to an equal competition of nanoscale mechanisms to accommodate strain during flow, holds true even at the strain rate of $10^{10} \mathrm{~s}^{-1}$.

The validity of this notion for the two high strain rates employed in MD simulations suggests generality for NC materials, but detailed studies at much lower strain rates are needed for evaluation of this assertion. At strain rates more commonly observed in experiments, the strongest grain size regime might shift to a smaller value, due to increased contribution of intragranular plastic deformation mechanisms. However, the exact shift can only be determined from the amount of increase in dislocation/twinning activity at these small strain rates, something which is not quantified and out of scope of a MD study. It should also be noted here that any extrapolation in the value of strongest grain size from simulation to experimental strain rates must be done with extreme care because the contributions of individual deformation mechanisms might vary differently in disparate strain rate regimes. The strain rate sensitivity of the mechanical behavior of NC metals, studied at different strain rates in several experimental and modeling studies ${ }^{33,43,44,47}$, suggest that the changes in contribution of individual mechanisms might be less pronounced when strain rate decreases from $10^{8} \mathrm{~s}^{-1}$ to experimental strain rates in comparison to the changes observed in this 
study, when the strain rate was varied from $10^{8}$ to $10^{10} \mathrm{~s}^{-1}$. Therefore, the actual shift in the strongest grain size at experimental strain rates might be smaller than one would expect from the extrapolation of simulation results. Moreover, at very small strain rates $\left(<1 \mathrm{~s}^{-1}\right)$, typically used in experiments, the contribution of GB mechanisms may rise again, due to activation of diffusion assisted sliding/creep processes ${ }^{44,48}$. The strongest grain size can, thus, be expected to again shift to higher values at smallest of strain rates, where diffusion assisted GB mechanisms are active ${ }^{48}$. Thus, it might even be possible that the strongest grain size value at two drastically different strain rates are equal, as also proposed by Li et al. ${ }^{44}$.

With the caveats discussed above, we regard the present MD simulations as offering utility in uncovering fundamental trends regarding the complex deformation behavior of NC materials, as also noted in previous studies ${ }^{42}$. These trends in NC behavior obtained from atomistic simulations can provide valuable decision support for rationalizing the strongest grain size regime in NC materials. To present such an example and to further assess the broader validity of these findings regarding mechanistic competition and maximum strength, we next consider alloyed NC structures. The addition of alloying elements can fundamentally alter the role of individual mechanisms during deformation by changing their propensity to plastically deform. It has recently been demonstrated experimentally in NC alloys that segregation of solute atoms to NC GBs shifted the strongest grain size to smaller values ${ }^{20}$. It was attributed to improved GB stability arising from solute segregation. Here, we show quantitatively that interfacial solute segregation fundamentally alters the landscape of competing nanoscale deformation mechanisms during flow, thereby, shifting the strongest grain size regime in accordance with the mechanistic competition conjecture discussed above.

\section{Mechanical behavior of NC Ni-P}

The Ni-P alloy system is selected for this study, since it has been shown experimentally to have improved strength and stability than NC Ni due to segregation of $\mathrm{P}$ atoms to $\mathrm{Ni} \mathrm{GBs}{ }^{49,50}$. Alloyed NC structures were generated from the NC Ni structures using Monte Carlo (MC)/ MD simulations (see "Methods" section). In the alloyed configuration, $\mathrm{P}$ atoms were found to strongly segregate to NC Ni GBs as seen experimentally ${ }^{51}$. Alloyed structures were then subjected to uniaxial tension MD simulations at a strain rate of $10^{8} \mathrm{~s}^{-1}$. Figure 6 a shows the variation of flow stress of alloyed $\mathrm{NC} \mathrm{Ni-P}$ as a function of average grain size- NC Ni behavior at $10^{8} \mathrm{~s}^{-1}$ strain rate is also shown for comparison. The data shows that $\mathrm{Ni}-\mathrm{P}$ flow stress magnitudes are greater than those of pure $\mathrm{Ni}$ across all grain sizes, exhibiting the expected strengthening arising from $\mathrm{P}$ segregation to $\mathrm{Ni}$ GBs. The amount of grain growth was also found to be smaller in $\mathrm{Ni}-\mathrm{P}$, in which case, the average grain size increased by $0.32 \mathrm{~nm}$ and $0.12 \mathrm{~nm}$ for NC Ni structures with initial grain sizes of $6 \mathrm{~nm}$ and $20 \mathrm{~nm}$, respectively. These results indicate that $P$ segregation strengthens the NC Ni GBs against grain growth, that is perpendicular to the boundaries, and also against shear in parallel, as reflected by increase in the values of flow stress. Furthermore, the strongest grain size shifts from $12 \mathrm{~nm}$ in pure $\mathrm{Ni}$ to $10 \mathrm{~nm}$ in $\mathrm{Ni}-\mathrm{P}$.

Hardness values taken from experimental studies of $\mathrm{NC} \mathrm{Ni}^{52-55}$ and $\mathrm{Ni}-\mathrm{P}^{50,56-58}$ samples, are plotted as a function of grain size in Fig. $6 \mathrm{~b}$. If the hardness values are scaled down by a factor of three to facilitate a rough comparison with the flow stress values ${ }^{59}$, we find that the simulation results are higher in magnitude than experiments for both $\mathrm{Ni}$ and $\mathrm{Ni}-\mathrm{P}$ structures, typical of high strain rate employed in MD simulations ${ }^{42}$. The experimental and the modeling data can further differ in magnitude because of other artifacts of MD simulations; and specific aspects of experimental samples, such as grain size and orientation distributions, synthesis routes, pre-existing impurity and defect content, intrinsic residual stress, overall solute concentration, and inherent errors in experimental measurements ${ }^{60}$. However, since atomistic simulations are capable of revealing proper trends regarding NC mechanical behavior, we can still compare trends in the variation of flow stress and hardness data as a function of grain size and solute content, as obtained from the simulations and experiments, respectively.

The simulation and the experimental data reveal some identical trends: (1) flow stress and hardness values are higher in Ni-P than $\mathrm{Ni}$ across all grain sizes, and (2) maxima in flow stress and hardness values occur at smaller grain sizes in $\mathrm{Ni}-\mathrm{P}$ as compared to Ni. The similar trends noticed in the simulation and the experimental data lends further credence to the simulation results presented in this study. The shifting of strongest grain size regime to smaller values in $\mathrm{Ni}-\mathrm{P}$ is also predicted by the notion relating maximum strength to an equal competition of governing deformation mechanisms during flow. Since, alloying strengthens the GBs in NC Ni-P, the contribution of GB-based deformation mechanisms in accommodating strain during flow should decrease across all grain sizes and the transition between the contributions of intragranular and GB mechanisms should, therefore, occur at a lower grain size. In order to test the validity of this notion in the alloyed case, the relative strain contributions of underlying mechanisms in NC Ni-P are quantified.

The change in the competition/cooperation of deformation mechanisms in NC structures as a function of alloying and grain size is illustrated in Fig. 7. Specifically, Fig. 7a shows slices through deformed NC structures where individual grains are colored according to the ratio of strain accommodation in GBs to grain interior, computed from the kinematic metrics at $5 \%$ applied
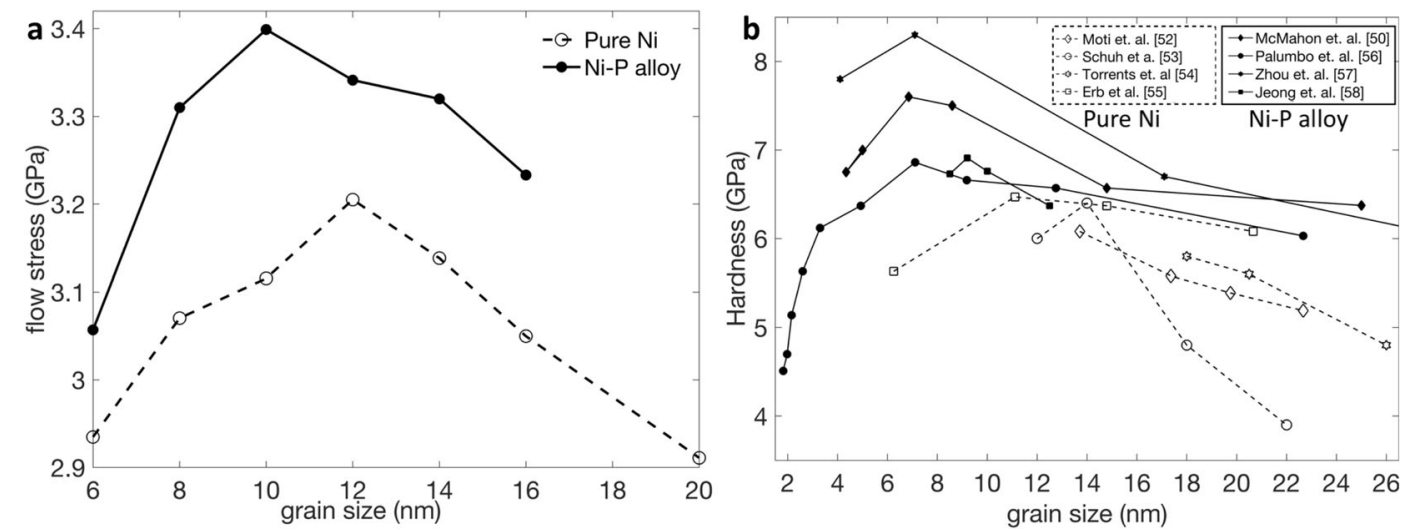

Fig. 6 Mechanical behavior of NC Ni-P. Variation of (a) flow stress and (b) hardness of NC Ni and Ni-P structures, as a function of average grain size, obtained from the uniaxial tension MD simulation and several past experimental studies, respectively. 


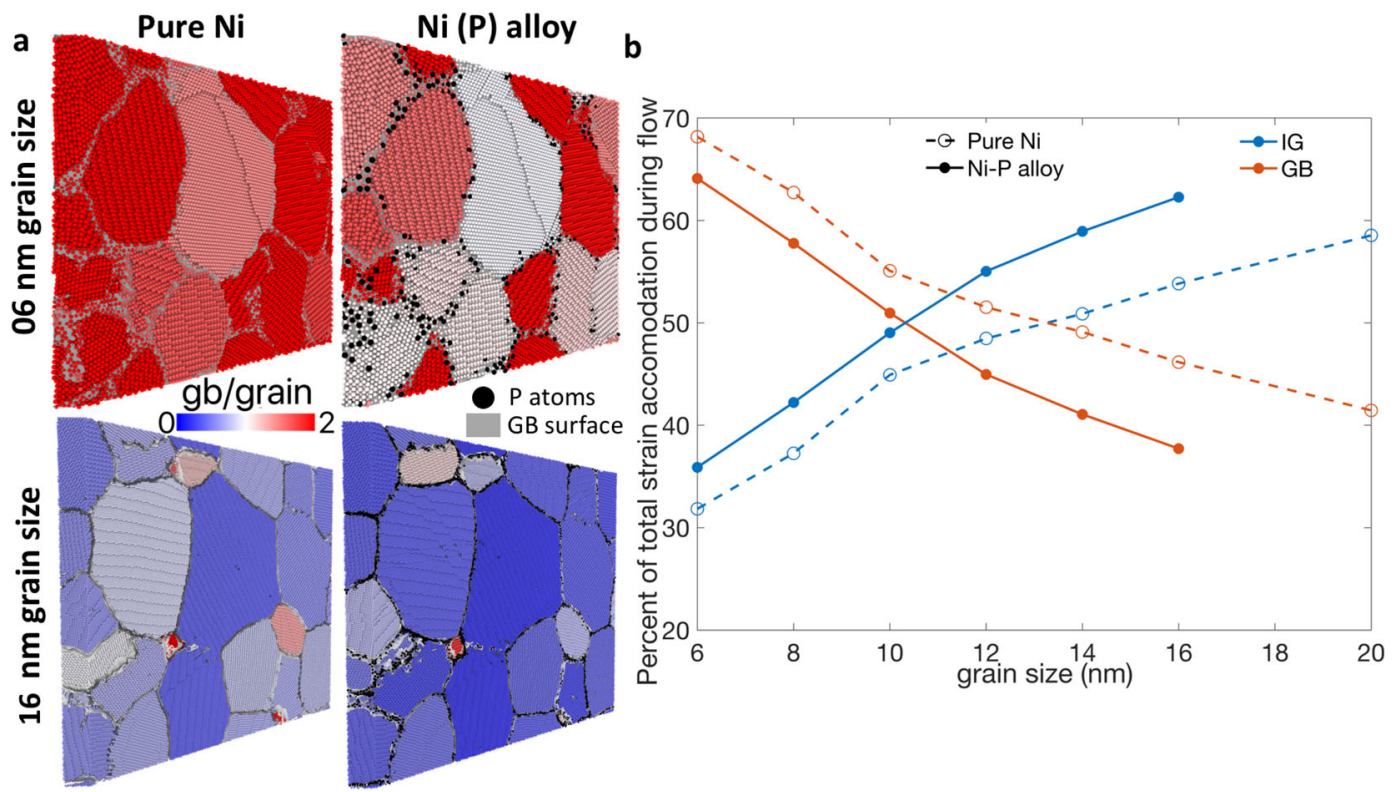

Fig. 7 Influence of alloying and grain size. (a) Slices through $6 \mathrm{~nm}$ and $16 \mathrm{~nm}$ grain size NC structures of Ni and Ni-P showing computed GB to grain interior strain accommodation ratio for individual grains at $5 \%$ applied strain. (b) Percentages of total strain accommodation by intragranular (IG) and intergranular (GB) mechanisms during flow in NC Ni and Ni-P structures, as a function of their average grain size.

strain. Strain within the majority of grains in NC Ni of $6 \mathrm{~nm}$ and $16 \mathrm{~nm}$ grain size is accommodated by intergranular (i.e., GB) and intragranular mechanisms, respectively. In Ni-P structures, the contribution of GB mechanisms decreases for both grain sizes, demonstrating the expected $G B$ strengthening arising from the presence of solute $\mathrm{P}$ atoms at NC Ni GBs. The influence of alloying and grain size on the overall competition of underlying nanoscale mechanisms is presented in Fig. 7b. The percentages of total strain accommodated within intragranular (IG) and GB mechanisms, averaged over the flow regime, are shown as a function of average grain size. Plots for individual grain sizes are shown in supplementary Fig. S3. The contribution of GB-based deformation mechanisms decreases while that of intragranular mechanisms increases in the alloyed case across all grain sizes. The results match well with the changes in the relative contributions of nanoscale mechanisms expected upon alloying, thereby, providing additional validation to both the MD simulations in capturing proper behavioral trends, and the kinematic metrics in accurately quantifying the simulated mechanical behavior. Furthermore, the transition between the two mechanisms happens at $10 \mathrm{~nm}$ grain

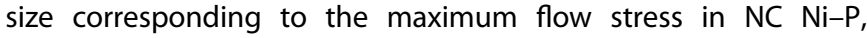
attesting that the strongest grain size still corresponds to an equal competition between grain-based and GB-based deformation mechanisms towards strain accommodation. Therefore, it can be established that the notion relating the maximum strength to an equal competition of nanoscale mechanisms holds true in the alloyed case as well.

In summary, we explicitly studied the notion relating the maximum strength to a transition in the governing deformation mechanisms from atomistic modeling by resolving the fraction of total strain accommodated by individual mechanisms using postprocessing kinematic metrics. It was found that in $\mathrm{NC} \mathrm{Ni}$, the maximum strength corresponds to the grain size regime where the governing mechanisms transition or equally compete to accommodate strain during flow, thus, verifying the notion. However, dislocation plasticity was not found to be the sole governing deformation mechanism at all grain sizes in the hardening regime contrary to the current understanding. Instead, it was shown that the combined contributions of all intragranular mechanisms govern the response of NC materials in the hardening regime. Furthermore, the results show that the notion holds only for total strain accommodation and not necessarily for its elastic and plastic components. The notion held at two MD strain rates tested in this study, further highlighting the effects of higher strain rate on the overall mechanical response of NC materials and the contributions of underlying mechanisms. The behavioral trends revealed by the simulation data were found to be in good agreement with the trends observed in experimental data or reported in previous experimental and modeling studies. Such agreements add confidence in generalizing the findings of this simulation study.

Finally, the notion was verified in alloyed NC Ni-P, where, the strongest grain size was shown to shift to smaller values due to decreased contributions of GB mechanisms, as suggested in past studies. The observed transition in the strongest grain size in NC $\mathrm{Ni}-\mathrm{P}$, based on the insight gained through pure $\mathrm{Ni}$, suggests that the strongest grain size regime in NC materials may possibly be tuned in other $\mathrm{NC}$ alloys by controlling the underlying operative deformation mechanisms at the nanoscale. Alloying is just one of several methods that are traditionally utilized to alter the structure and properties of NC materials. With the knowledge provided in this study of how these structural changes might fundamentally affect mechanistic competition, processing and chemistry routes may be rationally selected to shift the strongest grain size in NC materials to values which can be manufactured and tailored to each application. It will although require significant research and testing in different alloys and loading conditions such as creep where different operative mechanisms are competing or governing.

\section{METHODS}

Generation of NC Ni structures

In this study, atomistic NC Ni structures of average grain size ranging from $6 \mathrm{~nm}$ to $24 \mathrm{~nm}$ were created from phase field generated voxelated microstructure using the method outlined by Gruber et al. ${ }^{61}$. NC structures obtained from the phase field simulations tend to be more physically representative of topologies of grains, GBs and triple junctions and produce grain size distributions driven by thermodynamics of grain growth $^{61}$. In order to compute statistically averaged mechanical behavior, five different instances of NC structures were created for every grain size. In 
a

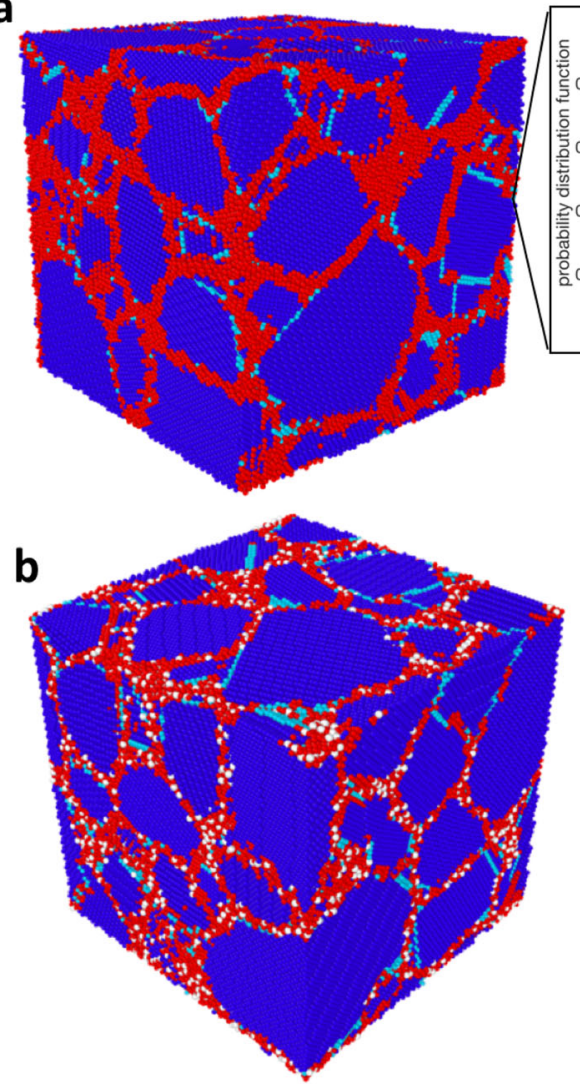

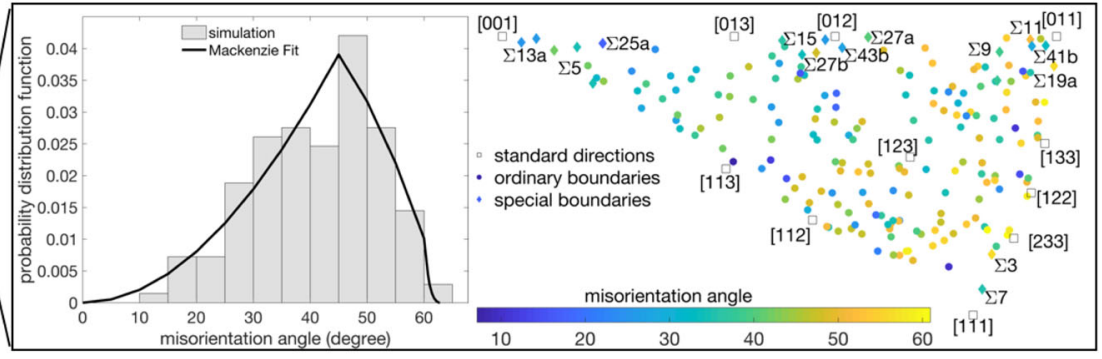

C

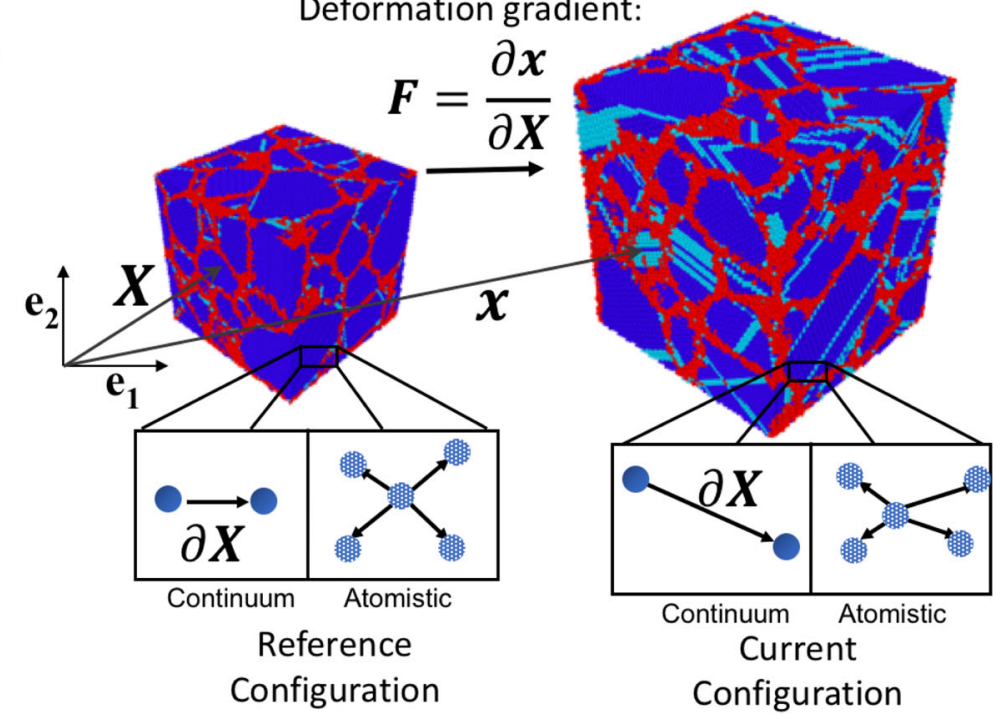

Fig. 8 Simulation Methodology. Atomistic NC structures of (a) Ni and (b) Ni-P of $6 \mathrm{~nm}$ grain average grain size (red: GB atoms, blue: FCC atoms, cyan: HCP atoms, white: P solute atoms; inset shows GB misorientation angle and axis distributions of NC Ni). (c) Schematic representation of the calculation of atomic deformation gradient tensor by approximating continuum material field around each atom using the nearest neighbor list.

each microstructural instance, the orientations of grains were chosen from different random distributions while employing identical microstructural topologies. Interatomic interactions in NC Ni structures are modeled using an embedded-atom-method (EAM) style potential ${ }^{62}$. This potential is parameterized to yield stacking fault energies similar to experimental values. Other key material properties such as lattice constant, phonon frequencies, vacancy formation energy were also fitted using ab initio calculations. All atomistic simulations in this study were performed using the LAMMPS software package ${ }^{63}$.

GBs in NC Ni structures were thermally equilibrated at high temperature $\left(0.5 \mathrm{~T}_{\mathrm{m}}\right)$ for $1 \mathrm{~ns}$ to reach local minima in energy rapidly ${ }^{64}$. Grain interior atoms were fixed during the high temperature equilibration to avoid grain growth; and the high temperature equilibration was followed by thermal equilibration of the whole system at $300 \mathrm{~K}$ with no constraint on any atoms. The equilibration was performed for an additional nanosecond, after which systems' potential energies became constant over time. The resulting structures had around 40 grains, and 3D periodic boundary conditions. The size of the simulation cells varied from $18 \mathrm{~nm}^{3}$ and 500,000 atoms at $6 \mathrm{~nm}$ grain size to around $72 \mathrm{~nm}^{3}$ and 35 million atoms at $24 \mathrm{~nm}$ grain size. Figure 8a shows a NC Ni structure of $6 \mathrm{~nm}$ average grain size along with the GB misorientation distribution. The GB misorientation angle distribution matches well with the Mackenzie distribution of a cubic sample $^{65}$, indicating a random texture. All visualizations in this work were performed using OVITO ${ }^{66}$.

\section{Generation of NC Ni-P structures}

Alloyed Ni-P structures were generated from NC Ni structures using hybrid $M C-M D$ simulations. A certain number of $P$ atoms (depending upon the desired solute content) were first placed at random inside NC bulk/ grains in-lieu of $\mathrm{Ni}$ atoms. Another EAM force field was used to model interatomic interactions in alloyed $\mathrm{Ni}-\mathrm{P}^{67}$. The potential was fitted to yield experimental lattice parameters and cohesive energies of $\mathrm{Ni}$ and $\mathrm{P}$. In addition, the potential was parametrized against a large ab initio database of energetics, lattice parameters and stress tensors of several configurations of Ni-P, including all intermetallic phases. Resulting NC structures were then subjected to hybrid MC-MD simulations. In the MC step, a pair of $\mathrm{P}$ and $\mathrm{Ni}$ atoms were selected at random and swapped by probabilities dictated by Metropolis criteria ${ }^{68}$ at $300 \mathrm{~K}$. Every $1000 \mathrm{MC}$ steps were followed by thermal relaxation of the entire system for $1000 \mathrm{MD}$ steps. Thermal equilibration was performed at $300 \mathrm{~K}$ using an isothermal-isobaric ensemble while maintaining zero normal pressure at periodic boundaries in all dimensions. The hybrid MC-MD simulation continued until the system's total potential energy reached a local minimum. In the final configuration, $\mathrm{P}$ atoms segregated to NC Ni GBs across all grain sizes in agreement with experiments ${ }^{49}$. An in-depth analysis of the interfacial solute segregation behavior in NC Ni-P as a function of GB character is provided in our recent investigation ${ }^{51}$. The bulk solute contents were chosen such that the total extent of solute segregation at GBs, or the GB solute content, was 12 atomic \% across all grain sizes. It should be noted that because of the large computational cost of hybrid MC-MD simulations, only one instance of the microstructure for each grain size is alloyed and mechanically tested. An alloyed configuration of NC Ni-P structure of $6 \mathrm{~nm}$ grain size is shown in Fig. $8 \mathrm{~b}$, where, segregation of $\mathrm{P}$ atoms at NC GBs is clearly visible.

\section{Mechanical testing}

Following the generation of NC structures, uniaxial tension MD simulations were performed under isothermal-isobaric conditions (NPT ensemble), with a constant imposed strain rate at $300 \mathrm{~K}$ temperature and zero normal pressure along boundaries in transverse directions. NC Ni structures were loaded at strain rates of $10^{8} \mathrm{~s}^{-1}$ and $10^{10} \mathrm{~s}^{-1}, \mathrm{Ni}-\mathrm{P}$ at $10^{8} \mathrm{~s}^{-1}$. Global pressure tensor of the system is used to calculate stress during uniaxial loading. In order to delineate elastic and plastic components of strain during loading, deformed NC structures were relaxed to drive system's 
elastic strain to zero. To enable a simulation restart from any point during the loading, NC structural configurations (position and velocities of atoms) at increments of $1 \%$ strain were stored in restart files. These configurations were then dynamically relaxed to a state of zero normal stress by applying a constant strain in the direction opposite to initial loading. The plastic strain was calculated from the atomic strain tensors in these relaxed structures. Only one instance of NC structure at a given grain size was subjected to these relaxation simulations due to large computational cost associated.

\section{Quantification of deformation mechanisms}

Strain tensors for every atom in the NC structure were calculated using the method described by Tucker et al. ${ }^{26,28,40,41}$. The deformation gradient tensor $\mathbf{F}$ was obtained by mapping the undeformed or reference configuration to a current or deformed configuration of $\mathrm{NC}$ structure as shown in Fig. 8c. Here, $\mathbf{x}$ and $\mathbf{X}$ are the vectors between two material points in the deformed and reference configurations, respectively. However, atomic positions instead of material points were used for this mapping in the atomistic structure, as detailed by Zimmerman et al. ${ }^{69}$. The deformation gradient tensor was estimated for each atom by mapping the nearest neighbor vectors of that atom in the reference and deformed configurations, as illustrated Fig. 8c. The strain tensor $\mathbf{E}$ was calculated from the deformation gradient tensor as, $\mathbf{E}=0.5\left(\mathbf{F}^{\top} \mathbf{F}-\mathbf{I}\right)$. Here, I is the identity tensor. Nanoscale deformation mechanisms were identified from the kinematic signatures of deformation around each atom, their structural environments ( $\mathrm{FCC} / \mathrm{BCC} / \mathrm{HCP} / \mathrm{Other})$ and slip vectors ${ }^{26,39,70}$. Particular identification of mechanisms (e.g., dislocation slip, twinning, GB deformation and elastic lattice strain) from computed atomic tensors was crucial. Based up on which, the percentage of total loading strain $p^{k}$, accommodated within a deformation mechanism $k$, at any point during the uniaxial loading was calculated according to Eq. 1.

$p^{k}=\frac{\sum_{i=1}^{n^{k}} E_{33}^{i}}{\sum_{i=1}^{N} E_{33}^{i}}$

Here, $E_{33}^{i}$ is the normal component along the loading direction ( $z$ in this case) of the strain tensor of an atom $i$ computed at that point during the uniaxial loading. Summations in the numerator and the denominator span over all atoms $n^{k}$, participating in the mechanism $k$, and the total number of atoms in the NC structure $N$, respectively. It should be noted that this method includes contribution from all atoms that were deformed by either dislocations, twins or GBs in previous configurations as well, not just those that are part of these defect structures in current configuration, and thus more accurately capturing the complex deformation that occurs within microstructures by competing mechanisms.

\section{DATA AVAILABILITY}

All data is available within the paper and supplementary information.

\section{CODE AVAILABILITY}

LAMMPS is an open-source atomistic simulation code, freely available to download at https://lammps.sandia.gov. All post-processing code and meta-data used in the analysis will be provided upon request.

Received: 13 May 2020; Accepted: 24 September 2020;

Published online: 14 October 2020

\section{REFERENCES}

1. Hall, E. O. The deformation and ageing of mild steel: Ill discussion of results. Proc. Phys. Soc. Sect. B 64, 747-753 (1951).

2. Petch, N. J. The cleavage strength of polycrystals. J. Iron Steel Inst. 174, 25-28 (1953).

3. Malow, T. R. \& Koch, C. C. Mechanical properties in tension of mechanically attrited nanocrystalline iron by the use of the miniaturized disk bend test. Acta Mater. 46, 6459-6473 (1998).

4. Conrad, H. Grain-size dependence of the flow stress of $\mathrm{Cu}$ from millimeters to nanometers. Metall. Mater. Trans. A 35, 2681-2695 (2004)
5. Hughes, G. D., Smith, S. D., Pande, C. S., Johnson, H. R. \& Armstrong, R. W. Hallpetch strengthening for the microhardness of twelve nanometer grain diameter electrodeposited nickel. Scr. Metall. 20, 93-97 (1986).

6. Baldwin, W. M. Yield strength of metals as a function of grain size. Acta Metall. $\mathbf{6}$ 139-141 (1958)

7. Dunstan, D. J. \& Bushby, A. J. Grain size dependence of the strength of metals: The Hall-Petch effect does not scale as the inverse square root of grain size. Int. J. Plast. 53, 56-65 (2014).

8. Schiøtz, J., Di Tolla, F. D. \& Jacobsen, K. W. Softening of nanocrystalline metals at very small grain sizes. Nature 391, 561-563 (1998).

9. Schiøtz, J. \& Jacobsen, K. W. A maximum in the strength of nanocrystalline copper. Science 301, 1357-1359 (2003).

10. Chokshi, A. H., Rosen, A., Karch, J. \& Gleiter, H. On the validity of the hall-petch relationship in nanocrystalline materials. Scr. Metall. 23, 1679-1683 (1989).

11. Fougere, G. E., Weertman, J. R., Siegel, R. W. \& Kim, S. Grain-size dependent hardening and softening of nanocrystalline $\mathrm{Cu}$ and Pd. Scr. Metall. Mater. 26, 1879-1883 (1992).

12. Konstantinidis, D. A. \& Aifantis, E. C. On the "Anomalous" hardness of nanocrystalline materials. Nanostruct. Mater. 10, 1111-1118 (1998).

13. Carlton, C. E. \& Ferreira, P. J. What is behind the inverse Hall-Petch effect in nanocrystalline materials? Acta Mater. 55, 3749-3756 (2007).

14. Lu, K. \& Sui, M. L. An explanation to the abnormal Hall-Petch relation in nanocrystalline materials. Scr. Metall. Mater. 28, 1465-1470 (1993)

15. Conrad, H. \& Narayan, J. On the grain size softening in nanocrystalline materials. Scr. Mater. 42, 1025-1030 (2000).

16. Lu, L., Shen, Y., Chen, X., Qian, L. \& Lu, K. Ultrahigh strength and high electrical conductivity in copper. Science 304, 422-426 (2004).

17. Yamakov, V., Wolf, D., Phillpot, S. R., Mukherjee, A. K. \& Gleiter, H. Deformation mechanism crossover and mechanical behaviour in nanocrystalline materials. Philos. Mag. Lett. 83, 385-393 (2003).

18. Lu, L., Chen, X., Huang, X. \& Lu, K. Revealing the maximum strength in nanotwinned copper. Science 323, 607-610 (2009).

19. Yamakov, V., Wolf, D., Phillpot, S. R., Mukherjee, A. K. \& Gleiter, H. Deformationmechanism map for nanocrystalline metals by molecular-dynamics simulation. Nat. Mater. 3, 43-47 (2004).

20. Hu, J., Shi, Y. N., Sauvage, X., Sha, G. \& Lu, K. Grain boundary stability governs hardening and softening in extremely fine nanograined metals. Science 355, 1292-1296 (2017).

21. Chandross, M. \& Argibay, N. Ultimate strength of metals. Phys. Rev. Lett. 124 125501 (2020).

22. Spearot, D. E., Tucker, G. J., Gupta, A. \& Thompson, G. B. Mechanical properties of stabilized nanocrystalline FCC metals. J. Appl. Phys. 126, 110901 (2019).

23. Hahn, E. N. \& Meyers, M. A. Grain-size dependent mechanical behavior of nanocrystalline metals. Mater. Sci. Eng. A 646, 101-134 (2015).

24. Zhang, L., Lu, C. \& Tieu, K. A review on atomistic simulation of grain boundary behaviors in face-centered cubic metals. Comput. Mater. Sci. 118, 180-191 (2016).

25. Stukowski, A., Albe, K. \& Farkas, D. Nanotwinned fcc metals: strengthening versus softening mechanisms. Phys. Rev. B 82, 224103 (2010).

26. Tucker, G. J., Tiwari, S., Zimmerman, J. A. \& McDowell, D. L. Investigating the deformation of nanocrystalline copper with microscale kinematic metrics and molecular dynamics. J. Mech. Phys. Solids 60, 471-486 (2012).

27. Prakash, A., Weygand, D. \& Bitzek, E. Influence of grain boundary structure and topology on the plastic deformation of nanocrystalline aluminum as studied by atomistic simulations. Int. J. Plast. 97, 107-125 (2017).

28. Tucker, G. J., Zimmerman, J. A. \& McDowell, D. L. Continuum metrics for deformation and microrotation from atomistic simulations: application to grain boundaries. Int. J. Eng. Sci. 49, 1424-1434 (2011).

29. Bitzek, E., Derlet, P. M., Anderson, P. M. \& Van Swygenhoven, H. The stress-strain response of nanocrystalline metals: a statistical analysis of atomistic simulations. Acta Mater. 56, 4846-4857 (2008).

30. Trelewicz, J. R. \& Schuh, C. A. The Hall-Petch breakdown in nanocrystalline metals: a crossover to glass-like deformation. Acta Mater. 55, 5948-5958 (2007).

31. Trelewicz, J. R. \& Schuh, C. A. Hot nanoindentation of nanocrystalline Ni-W alloys. Scr. Mater. 61, 1056-1059 (2009).

32. Liu, J., Fan, X., Zheng, W., Singh, D. J. \& Shi, Y. Nanocrystalline gold with small size: inverse Hall-Petch between mixed regime and super-soft regime. Philos. Mag. $\mathbf{0}$, 1-17 (2020).

33. Vo, N. Q., Averback, R. S., Bellon, P., Odunuga, S. \& Caro, A. Quantitative description of plastic deformation in nanocrystalline $\mathrm{Cu}$ : Dislocation glide versus grain boundary sliding. Phys. Rev. B. 77, 134108 (2008).

34. Kim, H. S. \& Bush, M. B. The effects of grain size and porosity on the elastic modulus of nanocrystalline materials. Nanostruct. Mater. 11, 361-367 (1999).

35. Valat-Villain, P., Durinck, J. \& Renault, P. O. Grain size dependence of elastic moduli in nanocrystalline tungsten. J. Nanomater. 2017, 1-6 (2017). 
36. Rajaram, S. S. et al. Grain-size-dependent grain boundary deformation during yielding in nanocrystalline materials using atomistic simulations. JOM 72, 1745-1754 (2020).

37. Maaß, R. \& Derlet, P. M. Micro-plasticity and recent insights from intermittent and small-scale plasticity. Acta Mater. 143, 338-363 (2018).

38. Van Swygenhoven, $\mathrm{H}$. Footprints of plastic deformation in nanocrystalline metals. Mater. Sci. Eng. A 483-484, 33-39 (2008).

39. Tucker, G. J., Foley, D. \& Gruber, J. Continuum metrics for atomistic simulation analysis. In Springer Series in Materials Science Vol. 245, 297-315 (Springer Verlag, 2016).

40. Tucker, G. J. \& Foiles, S. M. Quantifying the influence of twin boundaries on the deformation of nanocrystalline copper using atomistic simulations. Int. J. Plast. 65, 191-205 (2015).

41. Tucker, G. J., Zimmerman, J. A. \& McDowell, D. L. Shear deformation kinematics of bicrystalline grain boundaries in atomistic simulations. Model. Simul. Mater. Sci. Eng. 18, 015002 (2010).

42. Wolf, D., Yamakov, V., Phillpot, S. R., Mukherjee, A. \& Gleiter, H. Deformation of nanocrystalline materials by molecular-dynamics simulation: relationship to experiments? Acta Mater. 53, 1-40 (2005).

43. Rida, A., Micoulaut, M., Rouhaud, E. \& Makke, A. Understanding the strain rate sensitivity of nanocrystalline copper using molecular dynamics simulations. Comput. Mater. Sci. 172, 109294 (2020).

44. $\mathrm{Li}, \mathrm{H}$. et al. Mapping the strain-rate and grain-size dependence of deformation behaviors in nanocrystalline face-centered-cubic $\mathrm{Ni}$ and Ni-based alloys. J. Alloy. Compd. 709, 566-574 (2017).

45. Asaro, R. J. \& Suresh, S. Mechanistic models for the activation volume and rate sensitivity in metals with nanocrystalline grains and nano-scale twins. Acta Mater. 53, 3369-3382 (2005).

46. Wang, Y. M., Hamza, A. V. \& Ma, E. Temperature-dependent strain rate sensitivity and activation volume of nanocrystalline Ni. Acta Mater. 54, 2715-2726 (2006).

47. Brandl, C., Derlet, P. M. \& Van Swygenhoven, H. Strain rates in molecular dynamics simulations of nanocrystalline metals. Philos. Mag. 89, 3465-3475 (2009).

48. Mercier, S., Molinari, A. \& Estrin, Y. Grain size dependence of strength of nanocrystalline materials as exemplified by copper: An elastic-viscoplastic modelling approach. J. Mater. Sci. 42, 1455-1465 (2007).

49. Hentschel, T., Isheim, D., Kirchheim, R., Müller, F. \& Kreye, H. Nanocrystalline $\mathrm{Ni}-3.6$ at.\% $\mathrm{P}$ and its transformation sequence studied by atom-probe field-ion microscopy. Acta Mater. 48, 933-941 (2000).

50. McMahon, G. \& Erb, U. Bulk amorphous and nanocrystalline Ni-P alloys by electroplating. Microstruct. Sci. 17, 447-457 (1989).

51. Gupta, A., Zhou, X., Thompson, G. B. \& Tucker, G. J. Role of grain boundary character and its evolution on interfacial solute segregation behavior in nanocrystalline Ni-P. Acta Mater. 190, 113-123 (2020).

52. Moti, E., Shariat, M. H. \& Bahrololoom, M. E. Electrodeposition of nanocrystalline nickel by using rotating cylindrical electrodes. Mater. Chem. Phys. 111, 469-474 (2008).

53. Schuh, C. A., Nieh, T. G. \& Yamasaki, T. Hall-Petch breakdown manifested in abrasive wear resistance of nanocrystalline nickel. Scr. Mater. 46, 735-740 (2002).

54. Torrents, A., Yang, H. \& Mohamed, F. A. Effect of annealing on hardness and the modulus of elasticity in bulk nanocrystalline nickel. Metall. Mater. Trans. A 41, 621-630 (2010)

55. Erb, U. Electrodeposited nanocrystals: synthesis, properties and industrial applications. Nanostruct. Mater. 6, 533-538 (1995).

56. Palumbo, G., Erb, U. \& Aust, K. T. Triple line disclination effects on the mechanical behaviour of materials. Scr. Metall. Mater. 24, 2347-2350 (1990).

57. Zhou, Y., Erb, U., Aust, K. \& Palumbo, G. The effects of triple junctions and grain boundaries on hardness and Young's modulus in nanostructured Ni-P. Scr. Mater. 48, 825-830 (2003).

58. Jeong, D., Erb, U., Aust, K. \& Palumbo, G. The relationship between hardness and abrasive wear resistance of electrodeposited nanocrystalline Ni-P coatings. Scr. Mater. 48, 1067-1072 (2003)

59. Gouldstone, A. et al. Indentation across size scales and disciplines: recent developments in experimentation and modeling. Acta Mater. 55, 4015-4039 (2007).

60. Naik, S. N. \& Walley, S. M. The Hall-Petch and inverse Hall-Petch relations and the hardness of nanocrystalline metals. J. Mater. Sci. 55, 2661-2681 (2020).

61. Gruber, J., Lim, H., Abdeljawad, F., Foiles, S. \& Tucker, G. J. Development of physically based atomistic microstructures: the effect on the mechanical response of polycrystals. Comput. Mater. Sci. 128, 29-36 (2017).

62. Foiles, S. M. \& Hoyt, J. J. Computation of grain boundary stiffness and mobility from boundary fluctuations. Acta Mater. 54, 3351-3357 (2006).
63. Plimpton, S. Fast parallel algorithms for short-range molecular dynamics. J. Comput. Phys. 117, 1-19 (1995).

64. Tucker, G. J. \& Foiles, S. M. Molecular dynamics simulations of rate-dependent grain growth during the surface indentation of nanocrystalline nickel. Mater. Sci. Eng. A 571, 207-214 (2013).

65. Mackenzie, J. K. Second paper on statistics associated with the random disorientation of cubes. Biometrika 45, 229-240 (1958).

66. Stukowski, A. Visualization and analysis of atomistic simulation data with OVITO-the open visualization tool. Model. Simul. Mater. Sci. Eng. 18, 015012 (2010).

67. Sheng, H. W., Ma, E. \& Kramer, M. J. Relating dynamic properties to atomic structure in metallic glasses. JOM 64, 856-881 (2012).

68. Metropolis, N., Rosenbluth, A. W., Rosenbluth, M. N., Teller, A. H. \& Teller, E. Equation of state calculations by fast computing machines. J. Chem. Phys. 21, 1087-1092 (1953).

69. Zimmerman, J. A., Bammann, D. J. \& Gao, H. Deformation gradients for continuum mechanical analysis of atomistic simulations. Int. J. Solids Struct. 46, 238-253 (2009).

70. Zimmerman, J. A., Kelchner, C. L., Klein, P. A., Hamilton, J. C. \& Foiles, S. M. Surface step effects on nanoindentation. Phys. Rev. Lett. 87, 165507 (2001).

\section{ACKNOWLEDGEMENTS}

Authors G.J.T, G.B.T., and A.G. are grateful for the support of ARO through grant no. W911NF-17-1-0528 and greatly acknowledge ARO Program Manager, Dr. Michael Bakas. G.J.T. and A.G. also acknowledge XSEDE allocation charge no. TG-DMR190011 and $\mathrm{hpc}$ resource mio at Colorado School of Mines for allowing the reported atomistic simulations. D.L.M. is grateful for the support of the NSF CMMI-1761553.

\section{AUTHOR CONTRIBUTIONS}

Author G.J.T. conceived the project. A.G. performed all simulations and calculations. A.G., J.G., and G.J.T. developed the microstructure generation, characterization and kinematic metric codes. A.G. and G.J.T. analyzed the calculated results. All authors were involved in discussing the calculated results. A.G. and G.J.T. drafted the figures and manuscript with input from all authors. All authors reviewed and approved the manuscript.

\section{COMPETING INTERESTS}

The authors declare no competing interests.

\section{ADDITIONAL INFORMATION}

Supplementary information is available for this paper at https://doi.org/10.1038/ s41524-020-00425-0.

Correspondence and requests for materials should be addressed to G.J.T.

Reprints and permission information is available at http://www.nature.com/ reprints

Publisher's note Springer Nature remains neutral with regard to jurisdictional claims in published maps and institutional affiliations.

Open Access This article is licensed under a Creative Commons Attribution 4.0 International License, which permits use, sharing, adaptation, distribution and reproduction in any medium or format, as long as you give appropriate credit to the original author(s) and the source, provide a link to the Creative Commons license, and indicate if changes were made. The images or other third party material in this article are included in the article's Creative Commons license, unless indicated otherwise in a credit line to the material. If material is not included in the article's Creative Commons license and your intended use is not permitted by statutory regulation or exceeds the permitted use, you will need to obtain permission directly from the copyright holder. To view a copy of this license, visit http://creativecommons. org/licenses/by/4.0/

(c) The Author(s) 2020 